P361 LONG-TERM PEDIATRIC HOSPITAL ADMISSIONS AT A LEVEL III PORTUGUESE HOSPITAL - WHAT IS OUR REALITY?

Vanessa Gorito*, Marta Pinheiro, Tiago Magalhães, Rita Curval, Ana Maia, Manuel Fontoura. Department of Pediatrics. Centro Materno Pediátrico, Centro Hospitalar e Universitário de São João, Oporto, Portugal

\subsection{6/archdischild-2019-epa.707}

Introduction and aim Level III hospitals are the last resource of health care, covering more complex patients. According to the literature, these patients are hospitalized more often and for longer. Long-term hospitalizations are defined as episodes whose hospitalization time is equal to or greater than the upper threshold of exception and lower than the maximum threshold of the DRG to which they belong. We aim to characterize long-term hospitalizations in 2017 at a Level III Hospital.

Methods Retrospective analysis of long-term admissions in 2017 at a Level III Hospital, including: age, number of hospitalizations, resources at the emergency service, average length of stay, presence of chronic disease, comorbidities and pediatric appointments, through consultation of electronic processes. Results In 2017 there were 1493 hospitalized children; 1240 $(83 \%)$ admissions had a normal length of stay, $177(11.9 \%)$ were short admissions, 48 (3.2\%) long-term admissions and $28(1.9 \%)$ had prolonged evolution. We identified 49 patients with long-term hospitalizations. Of these, 33 males, mean age of 2.3 years ( $\min 1$ day, $\max 17$ years). Most were patients with chronic pathology (65.3\%), oncology (22.4\%), neurology $(16.3 \%)$ and neonatology (16.3\%). However, patients had, on average, 4 more diagnoses associated with the main reason for admission. Fever, respiratory or gastrointestinal symptoms prolonged or precipitated hospitalizations in $41.3 \%$ of patients. Ten of these patients had central venous catheter, 4 nasogastric tube/percutaneous gastrostomy, 2 ventricular shunts. On average, these patients had 2.79 hospital admissions per year, with an average length of hospital stay of 20.1 days and 1.69 appeals to the emergency department precipitated by sub-acute conditions such as gastroenteritis or respiratory diseases, which prolonged hospitalizations. In 15\% there were infections by multi-resistant microorganisms. The follow-up in multidisciplinary pediatric appointment occurred in $79.5 \%$.

Discussion Long-term admissions occurred mainly in chronically ill patients. Most, cancer or neurological patients, for decompensation of underlying disease. The percentage of patients infected with multi-resistant micro-organisms points to the need to adapt physical and medical resources to provide better care. The complexity of these patients demands the contribution of different Pediatric subspecialties and is associated with a higher mortality risk than the majority of Pediatric Services, which makes the hospitalization of our Service a reference in the provision of care in chronic pediatric illness in Portugal.

\section{P362 DELAYED TYPE HYPERSENSITIVITY REACTIONS TO ALUMINIUM-ADSORBED VACCINES: A CASE SERIES}

${ }^{1}$ Eimear Kelly*, ${ }^{1}$ Ronan Leahy, ${ }^{2}$ Siobhan Connors, ${ }^{2}$ Louise Kyne, ${ }^{1}$ Michael McDermott, ${ }^{1}$ Veronika Dvorakova, ${ }^{1}$ Rosemarie Watson. 'Our Lady's Children's Hospital, Crumlin, Dublin, Ireland; ${ }^{2}$ Temple Street Children's University Hospital, Dublin, Ireland

10.1136/archdischild-2019-epa.708
Persistent pruritic subcutaneous nodules have been reported at the site of vaccination (vaccination granulomas) following the use of several aluminium-adsorbed vaccines. An incidence of $0.8-0.9 \%$ has been reported in the literature. Contact allergy to aluminium has been strongly associated with the presence of vaccination granulomas. During the aluminium adsorbed diphtheria-tetanus/acellular pertussis vaccine trials, hypersensitivity to aluminium was demonstrated in $77 \%$ of those with pruritic nodules. These nodules appear to be long-lasting but little is known regarding prognosis.

In this case series, we report twelve children who developed pruritic nodules at injection sites following vaccination and were referred to a tertiary paediatric Dermatology centre for assessment between 2010 and 2018. The median age at onset of symptoms was twelve (IQR: 6 - 19.5) months and the main presenting symptoms were pruritus in eight children (67\%) and pain in three children. Six out of the seven children tested in the series for contact allergy for aluminium were positive (86\%). One child was found to have developed cutaneous pseudolymphoma on biopsy, a potential adverse effect of vaccines containing aluminium hydroxide as an adjuvant. This is the first case described in a child following vaccination. Four of the eleven children had imaging studies, two of which were reported as venous malformations.

Conclusion Although an infrequent occurrence following vaccination with aluminium-adsorbed vaccines, the development of persistent pruritic nodules can cause significant distress and anxiety for parents and children and lead to unnecessary investigations and a delay in subsequent vaccination. Greater awareness among clinicians and primary health care providers of this potential adverse reaction is necessary.

\section{P363 THE UNDERGRADUATE EDUCATION ABOUT VACCINATION AND VACCINE HESITANCY}

\footnotetext{
${ }^{1,2}$ Irena Bralic*, ${ }^{3}$ Katija Pivalica. 'University of Split School of medicine, Split, Croatia; ${ }^{2}$ Specialist Department for Pediatrics, Trogir, Croatia; ${ }^{3}$ Pediatrics Clinic, University Hospital Centre, Split, Croatia
}

\subsection{6/archdischild-2019-epa.709}

Background We are witnessing the modern paradox about vaccinations and vaccines. Vaccinations are the best method to deal with, eliminate and eradicate preventable diseases. It's natural that the view on vaccines is different according to parents, doctors and media. As pediatricians, we always use the Convention of childrens' rights as the basis of our medical advice.

Aim In cross-sectional study was analysed the experience in undergraduate education about vaccination among $758 \mathrm{stu}-$ dents of medicine from $1^{\text {st }}$ to $6^{\text {th }}$ year, at the University of Split School of medicine.

Results Only 33\% of medical students believe that they are given sufficient information on vaccination and vaccines during their regular under-graduate studies. It is absurd that $40 \%$ of Croatian medical students, as future educators and promoters of vaccination, are being educated from professionally and scientifically unsubstantiated sources.

Discussion The health culture of prevention of disease by vaccination is a reflection of the enlightenment of the population, the accessibility of the health care system, and the development of society. An effort is made to include the vaccination programme, as a complex medical intervention, in the regular health care system. Vaccines are still not sufficiently available 
in underdeveloped countries due to poverty. At the same time, despite the obvious benefits of vaccination, especially in developed countries, there has been a noticeable rise in vaccine hesitancy. With the fall in the number of vaccinated people on a global, national, regional and local level, the risk is growing of epidemics breaking out once again of illness which until recently were in the phase of elimination.

Conclusion Evidence based education, especially the experience and attitudes of all health professionals, as potential educators of the population, is the foundation for successful vaccination. The responsibility of doctors for the implementation of vaccination programmes is great, regardless whether they are directly involved in their realization or not. For the sake of better preparation of future doctors for clinical practice and public health, it is vital to strengthen the quality in undergraduate education about vaccinationin order to overcome the problem of vaccine hesitancy.

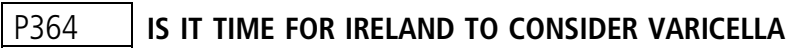 VACCINATION TO THE NATIONAL IMMUNIZATION PROGRAMME?}

1,2 Roy K Philip*, ${ }^{2}$ Trina Dooley, ${ }^{3}$ Janice Murtagh, ${ }^{4}$ Suzanne Cotter. ${ }^{1}$ Graduate entry Medical School, University of Limerick, Limerick, Ireland; ${ }^{2}$ University Hospital Limerick, Limerick, Ireland; ${ }^{3}$ Vaccines division, MSD Ireland Ltd., Dublin, Ireland; ${ }^{4}$ Health Protection Surveillance Centre (HPSC), Dublin, Ireland

\subsection{6/archdischild-2019-epa.710}

Background Varicella (chickenpox) is generally considered a mild illness; however the resultant disease burden is substantial. Uptake of the live-attenuated vaccine to National immunization programmes is variable among European countries. Varicella could pose serious illness and mortality among vulnerable populations such as immunocompromised and those with significant co-morbidities. Varicella vaccine is commercially available in Ireland, however not funded for universal immunization. Since 2012 hospital admissions with varicella is notifiable in Ireland.

Aims To analyse the reporting of hospitalised cases of varicella in Ireland from 2012 to 2018 for 0 to 19 years of age and to propose the potential benefits of inclusion varicella immunization to the National programme.

Methods Surveillance data submitted to health protection surveillance centre (HPSC) from January 2012 to December 2018 from hospitals around all the HSE regions of the country was analysed. Annualised rates for age categories of 0-4 years, 5-9 years, 10-14 years and 15-19 years were determined. Cross verification with hospital inpatient enquiry (HIPE) data was conducted; bed days consumed and length of stay (LOS) were estimated. Results were compared with previously published UK/Irish rates. Mortality was not analysed as part of the study. Approval for analysis of collated data from HPSC and HIPE was obtained.

Results There were 444 hospitalisations for the 0 to 19 years (mean of 63.4 admissions/year). $320 / 44$ (72\%) were in $0-4$ years and 94/444 (21\%) among 5-9 years, together contributing to $93 \%$ of hospitalisations. With a mean LOS of 2.86 days the varicella admissions contributed to 1269.8 bed days (inclusive of general wards, paediatric high dependency unit and paediatric intensive care unit bed utilisation) based on HIPE estimates. An increasing trend of hospitalisation was observed year-on-year for 0-4 and 5-9 years. HPSC reporting was comparable to, however lower than, the active British paediatric surveillance unit (BPSU) study published in 2007 (including Irish data). Our sentinel rate estimate of 133.1/ 100,000 population (range 98.8 -224.7) reflects community burden of varicella.

Conclusions Improving and standardising the varicella surveillance, highlighting the preventable acute hospital bed days due to serious illness from varicella, analysing the disease specific mortality, accurately estimating the disease burden in community including the societal costs and predicting the future implications to rate of herpes zoster among adults and the elderly; all should be factored-in to make a case for the inclusion of varicella to the National immunization programme in Ireland.

\section{P365 PERTUSSIS VACCINATION: SHOULD WE BE DOING SOMETHING DIFFERENT?}

Louise Hendrick* , Mary Ward. Department of Public Health, HSE East, Ireland

\subsection{6/archdischild-2019-epa.711}

Background Pertussis is a highly infectious disease and an important public health concern. The main aim of pertussis vaccination is to reduce the risk of severe pertussis in infants and young children, due to the high disease-related morbidity and mortality in this age group. While the introduction of whole-cell vaccine in the 1940s led to a dramatic decrease in cases and associated fatalities, its higher reactogenicity led to its replacement with acellular vaccines, which contain fewer antigens.(1) In Ireland, despite vaccination coverage rates of 95\% from 2011-2018 average rates from 2014-2018 were more than double those from 2003-2008.(2) Infants under 6 months and adults between 35 and 44 years have been disproportionately affected.(3)

Aim The aim of this review was to assess the effectiveness of wholecellular versus acellular pertussis; to analyse the relevance of recent trends in circulating strains including genetic divergent isolates; to assess the effectiveness of varying the number of antigen components in acellular vaccines and to determine the impact of different vaccination schedules on vaccine efficacy.

Methods A review of the literature was performed across three electronic databases from January 1990 to October 2018, using key search terms. A search of grey literature using the same terms and time period was also conducted.

Conclusion National Vaccination programs have not led to optimal pertussis control. Variations in scheduling or type of vaccine (wholecell or acellular) has not resulted in improved control. The change to acellular vaccination highlighted the relatively short-lived benefit on adaptive immunity and protection and lead to the introduction of booster vaccination doses at a younger age and at adolescence to counter waning immunity. Acellular vaccinations contain between one and five antigenic components. Vaccines which contain between three and five antigens demonstrate higher efficacy than vaccines with lower antigen components. In Denmark and Sweden effectiveness studies have shown some benefit in mono-component and two-antigen vaccines, although factors such as surveillance, diagnosis, variation in case definition and differences in uptake rates make comparison difficult. Mismatches between circulating strains and specific vaccine antigens, particularly pertactin, have been reported, although no evidence of higher virulence has been associated with these isolates. 